\title{
Lensless diffractive imaging with ultra-broadband table-top sources: from infrared to extreme-ultraviolet wavelengths
}

\begin{abstract}
Stefan Witte ${ }^{1}$, Vasco T Tenner ${ }^{1,2}$, Daniel WE Noom ${ }^{1}$ and Kjeld SE Eikema ${ }^{1}$
Lensless imaging is an approach to microscopy in which a high-resolution image of an object is reconstructed from one or more measured diffraction patterns, providing a solution in situations where the use of imaging optics is not possible. However, current lensless imaging methods are typically limited by the need for a light source with a narrow, stable and accurately known spectrum. We have developed a general approach to lensless imaging without spectral bandwidth limitations or sample requirements. We use two time-delayed coherent light pulses and show that scanning the pulse-to-pulse time delay allows the reconstruction of diffraction-limited images for all the spectral components in the pulse. In addition, we introduce an iterative phase retrieval algorithm that uses these spectrally resolved Fresnel diffraction patterns to obtain high-resolution images of complex extended objects. We demonstrate this two-pulse imaging method with octave-spanning visible light sources, in both transmission and reflection geometries, and with broadband extreme-ultraviolet radiation from a high-harmonic generation source. Our approach enables effective use of low-flux ultra-broadband sources, such as table-top high-harmonic generation systems, for high-resolution imaging.
\end{abstract}

Light: Science \& Applications (2014) 3, e163; doi:10.1038/lsa.2014.44; published online 28 March 2014

Keywords: coherent diffractive imaging; extreme ultraviolet; Fourier transform spectroscopy; high-harmonic generation; lensless imaging

\section{INTRODUCTION}

Lensless imaging is an elegant approach to high-resolution microscopy that is rapidly gaining popularity in applications where the use of imaging optics is problematic. This situation occurs, e.g. in imaging experiments using radiation at extremely short wavelengths, such as in the extreme-ultraviolet (XUV) and X-ray spectral ranges, for which the development of good-quality imaging optics is extremely complex. ${ }^{1,2}$ Another important application is in the development of miniaturized visible-light microscopes, to avoid the use of imaging optics to optimize the size and cost of the device. . $^{3,4}$

In lensless imaging, an object is illuminated by a coherent light source and the resulting diffraction pattern is recorded. An image of the object must then be reconstructed numerically; this can be performed by field propagation methods if both the intensity and phase of the diffraction pattern are known. As only the intensity is recorded in a typical diffraction measurement, the central issue in lensless imaging is the retrieval of phase information from a recorded diffraction pattern. Solutions to this problem have been found both through numerical and optical means. ${ }^{1,5,6}$ In coherent diffractive imaging, the missing phase information is numerically reconstructed by iterative algorithms, ${ }^{7}$ a process that has been shown to have a unique solution as long as the oversampling condition is satisfied. ${ }^{8}$ This condition can be satisfied by using a finite object support, ${ }^{9,10}$ a localized illumination profile $^{11}$ and/or recording multiple partially overlapping diffraction patterns. ${ }^{12,13}$ Holographic methods, which directly record the phase through interference with a separate reference wave, have also been developed for a lensless imaging context. ${ }^{6,14}$

There is a growing need for lensless imaging methods that operate with broadband radiation, to make much more efficient use of such state-of-the-art light sources as third generation synchrotrons and table-top high-harmonic generation (HHG) sources for imaging. In particular, HHG-based sources hold promise for compact and costeffective ultrahigh-resolution microscopy and recent breakthroughs have led to the generation of a significant photon flux in the biologically important water-window spectral range. ${ }^{15,16}$ However, HHG sources intrinsically produce an ultra-broadband soft-X-ray spectrum, and the need to spectrally filter these sources leads to a reduction in flux of 3-4 orders of magnitude, which severely limits their use for imaging applications. Several pioneering proof-of-concept experiments have already been performed with table-top HHG sources ${ }^{17-22}$ and the ability to use the full flux of such a source for imaging could provide a breakthrough for practical imaging applications.

In general, the use of monochromatic radiation has always been a major requirement for any diffractive imaging experiment, as the

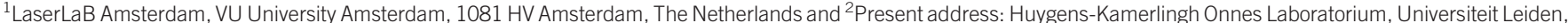
2333 CA Leiden, The Netherlands

Correspondence: Dr S Witte, LaserLaB Amsterdam, VU University Amsterdam, De Boelelaan 1081, 1081 HV Amsterdam, The Netherlands

E-mail: s.m.witte@vu.nl

Received 8 October 2013; revised 8 January 2014; accepted 9 January 2014 
angle at which light diffracts from any structure depends on its wavelength. A diffraction pattern generated by a polychromatic source will therefore consist of a superposition of the diffraction patterns of all the individual spectral components. ${ }^{23}$ Depending on the spectral bandwidth of the source, this effect limits the achievable resolution ${ }^{24}$ and for more broadband sources, can inhibit image reconstruction entirely. By incorporating knowledge of the source spectrum into the reconstruction algorithm, discrete $\operatorname{spectra}^{23}$ and finite bandwidths ${ }^{25}$ can be handled. However, such calculations require a stable, accurately calibrated source spectrum and therefore, remains limited to relatively narrow spectral bandwidths $(\Delta \lambda / \lambda \approx 0.028$ has been shown ${ }^{25}$ ).

We demonstrate that all bandwidth limitations on lensless imaging can be removed by the illumination of the sample with two coherent time-delayed pulses (Figure 1a). If a series of diffraction patterns is recorded as a function of the time delay between the two pulses, methods from Fourier transform spectroscopy ${ }^{26}$ can be used to obtain spectrally resolved information from the recorded diffraction patterns themselves. No additional measurement of the source spectrum is required, and the spectrum may be ultra-broadband, highly structured and even exhibit significant intensity fluctuations (see the Supplementary Information for detailed simulations). Our two-pulse imaging method is universal and can be applied to any diffractive imaging experiment. In the following sections, we illustrate this with experimental demonstrations for both Fresnel and far-field diffraction, for transmission and reflection geometries and for diffractive imaging using an XUV HHG source.

\section{MATERIALS AND METHODS}

\section{Two-pulse imaging with an ultra-broadband source}

When performing lensless imaging with an ultra-broadband source, the recorded ultra-broadband diffraction patterns can be interpreted as a linear superposition of monochromatic diffraction patterns. Knowledge of the superposition coefficients would therefore enable the spectral decomposition of the diffraction patterns into their individual spectral components. In practice, this approach means that we need a way to measure the distribution of the spectral components that have scattered onto each camera pixel (see Supplementary Information and Supplementary Fig. S1 for details). The key point of our method is that the spectral decomposition of the diffraction patterns can be achieved by recording the scattering signal for each individual camera pixel as a function of the pulse-to-pulse time delay (Figure 1b). For each pixel, this delay time-dependent signal is equal to the Fourier transform of the spectrum of the light scattered onto that individual pixel. Therefore, a Fourier transform converts it back to the frequency domain and reveals the spectral components scattered onto each pixel (Figure 1c). With this information, spectrally resolved diffraction patterns can be reconstructed throughout the entire source spectrum from a single time delay scan (see Supplementary Information for details). The spectral resolution is inversely proportional to the scanned time delay, similar to Fourier transform spectroscopy. ${ }^{26}$ A powerful feature of this approach, common to many Fourier transform-based methods, ${ }^{26-28}$ is that the full-source spectrum is used throughout the entire measurement, resulting in an efficient use of the available photon flux.

\section{Two-pulse imaging in the Fraunhofer regime}

For the first experimental example of two-pulse imaging at visible wavelengths, we use a spatially coherent octave-spanning continuum as the light source. Two time-delayed pulses are generated in a scanning
Michelson interferometer and sent into a lensless imaging set-up (Supplementary Fig. S2). Coherent white-light continuum pulses are produced by launching ultrashort laser pulses into a photonic crystal fiber. A time-delayed pulse pair is produced using a Michelson interferometer, with one of the end mirrors mounted on a closed-loop piezo stage. The pulse pair is then used to illuminate a sample, and the diffracted light is recorded using a 14-bit CCD camera with $1936 \times 1456$ pixels and $4.54 \mu \mathrm{m}$ pixel size. We typically acquire 500 individual images with $0.01-1 \mathrm{~ms}$ exposure times, while increasing the time delay in $0.67 \mathrm{fs}(200 \mathrm{~nm})$ steps.

As a proof-of-concept experiment, we image the test sample shown in Figure 1d, which consists of some small structures that have been laser-etched in a gold layer deposited on a glass substrate. We achieve far-field conditions by positioning the CCD camera $5 \mathrm{~cm}$ behind an $f=5 \mathrm{~cm}$ lens located behind the sample, which ensures both a far-field imaging geometry and a moderate numerical aperture for visible wavelengths.

\section{RESULTS AND DISCUSSION}

The measured diffraction pattern using the full-source spectrum is shown in Figure 1e. A strong radial smearing is present: owing to the wavelength-dependent diffraction angle, each point in the diffraction pattern is convolved with a large spectral bandwidth in the radial dimension. However, from the two-pulse scan and the Fourier transformation, quasimonochromatic diffraction patterns are obtained (Figure 1f) in which the radial structure of the diffraction pattern is clearly resolved. The spectral bandwidth of these retrieved images is determined by the length of the two-pulse scan, and it can readily be made sufficiently small (by a longer delay scan) to treat the images as monochromatic. Figure $1 \mathrm{~g}$ shows an overlay of three diffraction patterns at different wavelengths, obtained from a single two-pulse scan. The dramatic improvement in image reconstruction is clearly visible from Figure $1 \mathrm{~h}-1 \mathrm{j}$, in which an iterative phase retrieval algorithm is applied to retrieve an image from the diffraction patterns in Figure 1e1g. For the phase retrieval, a support is defined from the autocorrelation of the diffraction pattern, after which 300 iterations of the error reduction algorithm ${ }^{10}$ are used with a random initial phase guess. The algorithm is run 20 times and the results are averaged into the final image. Because of the low coherence in the unresolved broadband diffraction pattern of Figure 1e, the requirements for applying Gerchberg-Saxton-type iterative phase retrieval algorithms are not satisfied and the resulting image (Figure $1 \mathrm{~h}$ ) is extremely poor. In stark contrast, the image reconstructed from the two-pulse scan (Figure 1i) is a high-quality image of the test object, with a diffraction-limited resolution of $6 \mu \mathrm{m}$ (limited by the numerical aperture of 0.046). Three reconstructions at different wavelengths, corresponding to the diffraction patterns shown in Figure 1g, are overlaid in Figure 1j. All the reconstructions provide a clear image of the test object, but their relative sizes are different due to the wavelength-dependent field of view discussed above. After appropriate scaling, all these images can be averaged to improve the final signal-to-noise ratio. In this case, the full-source spectrum is used efficiently for image reconstruction.

\section{Multiwavelength phase retrieval}

The spectrally resolved diffraction data also suggest new possibilities for fast and robust phase reconstruction, based on the propagation effects in coherent diffractive imaging in the Fresnel regime. In the far-field (Fraunhofer) regime discussed above, the only effect of propagation is a global scaling of the diffraction pattern. However, in the Fresnel regime, there is an additional propagation and wavelength-dependent 

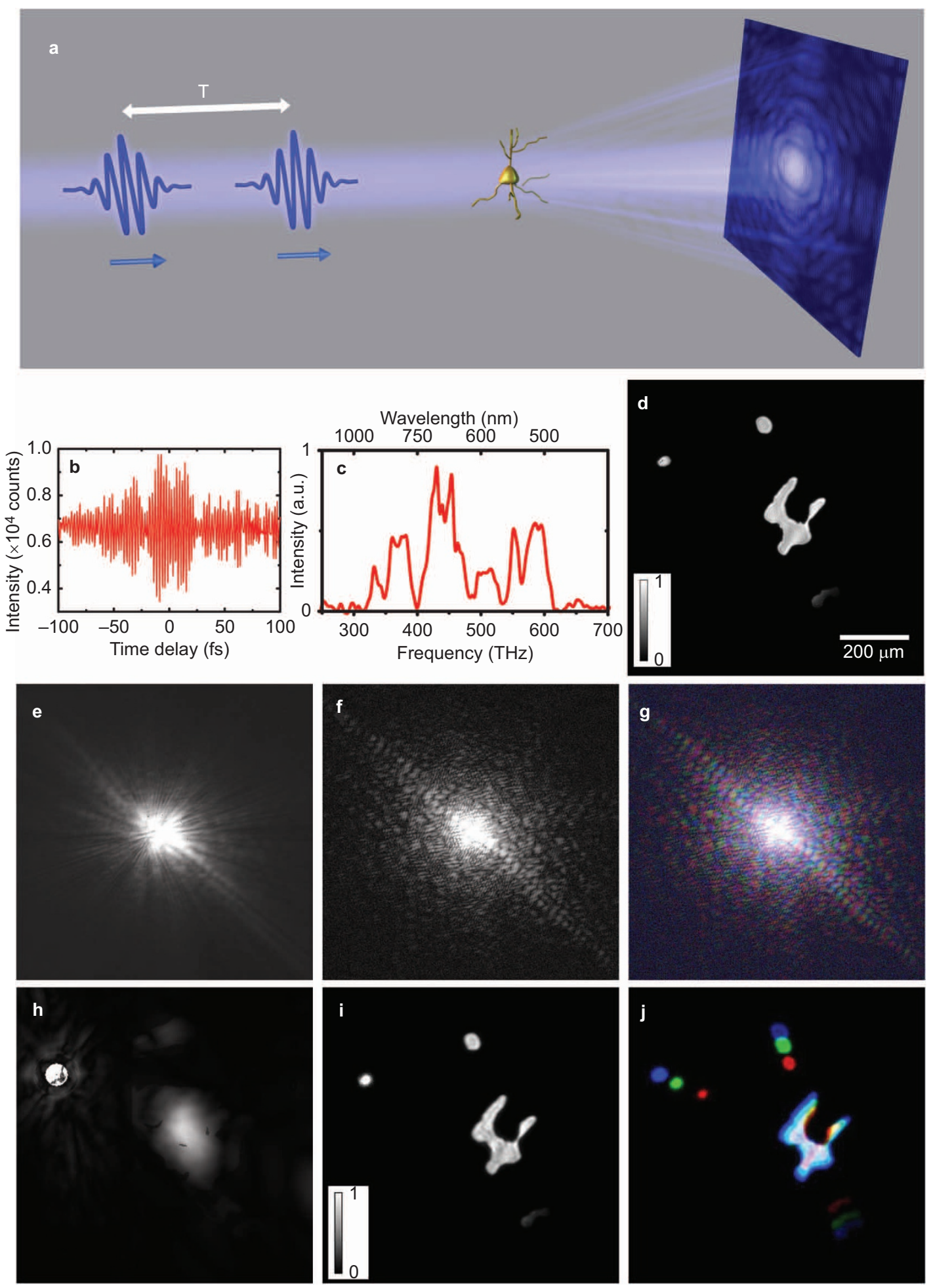

Figure 1 Principle of two-pulse imaging and experimental results in the Fraunhofer regime. (a) A sample is illuminated with two coherent pulses, and a set of diffraction patterns is recorded as a function of time delay $T$ in a lensless imaging geometry. (b) A typical signal recorded on a single pixel during a coherent diffractive imaging experiment using two pulses, as a function of time delay $T$. (c) Fourier transform of the signal in $\mathbf{b}$, showing the light spectrum diffracted onto that specific pixel. By reconstructing such spectra for all pixels, spectrally resolved diffraction patterns can be extracted throughout the full-source spectrum. (d) White-light microscope image of a laser-machined sample, containing transmitting structures in a gold layer. (e) Measured far-field diffraction pattern (log-scale) using the full-source spectrum. (f) Reconstructed diffraction pattern (log-scale) at $565 \mathrm{~nm}$ wavelength, retrieved from a two-pulse scan. (g) Overlay of three retrieved diffraction patterns (log-scale) at wavelengths of 508,565 and $694 \mathrm{~nm}$. (h) Result of iterative phase retrieval using the broadband diffraction pattern of e. No image can be retrieved due to the excessive spectral bandwidth. (i) Result of iterative phase retrieval using a diffraction pattern retrieved from the two-pulse scan (from f). (j) Overlay of three independent reconstructed images from the diffraction patterns in $\mathbf{g}$, which all show good-quality images of the sample. The field of view is wavelength-dependent, resulting in a different image size for each wavelength. 
spatial phase evolution. This propagation-dependent phase evolution can be exploited for more robust phase retrieval strategies, based on the knowledge of the wavefront curvature ${ }^{29-31}$ or by performing measurements at multiple sample-to-camera distances. ${ }^{32,33}$

We have developed a novel iterative phase retrieval scheme that explicitly uses the recorded multiwavelength data to reconstruct the phase without the need for support constraints. In the Fresnel regime, wave propagation couples the amplitude and phase of an electric field $E(x, y, z)$ through the Fresnel diffraction integral:

$$
E(x, y, z)=\frac{e^{i 2 \pi z / \lambda}}{i \lambda z} \iint E\left(x^{\prime}, y^{\prime}, 0\right) e^{(i \pi / \lambda z)\left[\left(x-x^{\prime}\right)^{2}+\left(y-y^{\prime}\right)^{2}\right]} \mathrm{d} x^{\prime} \mathrm{d} y^{\prime}
$$

where propagation is along the $z$-coordinate and $\lambda$ is the wavelength of the light. Equation (1) states that Fresnel propagation depends on the distance and wavelength in an identical way (aside from a global phase factor), allowing us to exploit our spectrally resolved diffraction data to 'propagate' between different spectral components, as schematically depicted in Figure 2a. This scheme does not require sample or camera movement and relies only on measured diffraction patterns at multiple wavelengths as input. It converges reliably and works for extended samples. Keyhole imaging ${ }^{11,34}$ and ptychography ${ }^{12,13}$ have also been used successfully to image parts of extended samples, but our multiwavelength approach does have the advantage that spatially confined illumination is not required for stable convergence.
To demonstrate this multiwavelength phase retrieval approach, we record two-pulse scans of Fresnel diffraction patterns of a fixed sample of Convallaria majalis (Lily of the Valley) rhizome, of which a broadband diffraction pattern is shown in Figure 2b. Figure 2c shows a narrow-band $(\Delta \lambda=5 \mathrm{~nm})$ diffraction pattern of this Convallaria sample at a wavelength of $695 \mathrm{~nm}$, which has been extracted from a twopulse imaging scan. Sharp diffraction features are clearly visible, whereas they were washed out in the broadband images.

For our multiwavelength Fresnel reconstruction method, phase retrieval is performed in a Gerchberg-Saxton-type iterative scheme, where the intensity data from the first wavelength are propagated to the next wavelength through the evaluation of the Fresnel propagation Equation (1). After propagation, the phase information is retained, while the intensity is replaced with the measured intensity at this new wavelength. A detailed description of the multiwavelength phase retrieval algorithm is provided in Supplementary Information. The choice of wavelengths to be used in the reconstruction is flexible: the main requirement is that they be sufficiently spectrally separated to display significant differences after propagation over a distance $z$, i.e., the phase term inside the integral in Equation (1) should change by a measurable amount between wavelengths for a detector pixel at position $(x, y)$. This phase shift condition depends on the detection geometry and is readily fulfilled in practice. We typically select $2-5$ images separated by $5-20 \mathrm{THz}$ in frequency from the spectrally resolved
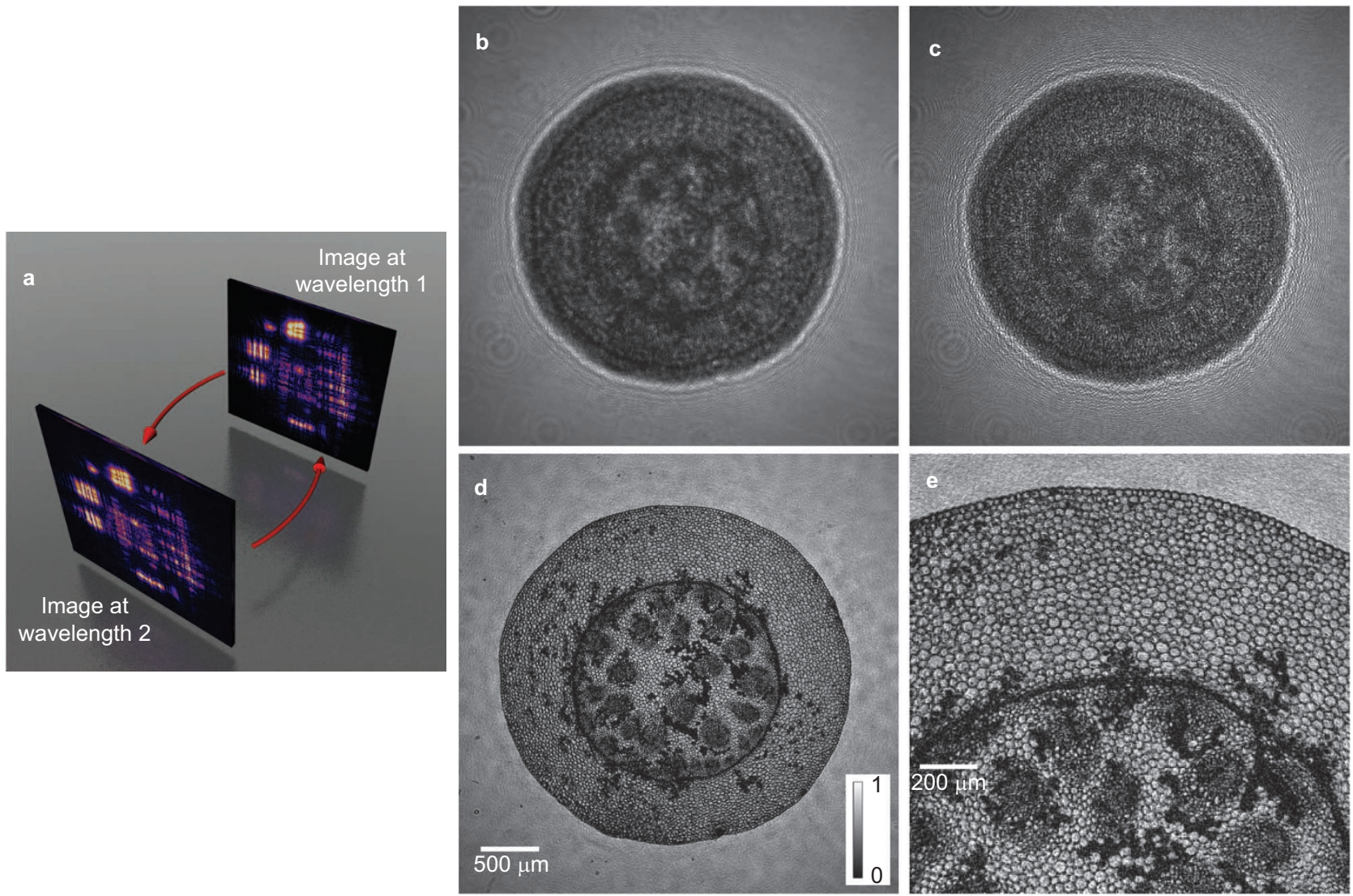

Figure 2 Multiwavelength phase retrieval in the Fresnel regime. (a) Graphic representation of the multiwavelength phase retrieval algorithm. Iterative propagation is performed between Fresnel diffraction patterns recorded at the same distance, but with different wavelengths. (b) Broadband Fresnel diffraction pattern of Convallaria majalis rhizome, recorded using an octave-wide visible light source. (c) Quasimonochromatic Fresnel diffraction pattern of the same Convallaria sample as in b, obtained from a two-pulse imaging scan. Much finer diffraction features are visible. (d) Resulting image after 25 iterations of multiwavelength iterative phase retrieval, using diffraction patterns at five different wavelengths. (e) Close-up of a part of the reconstructed image, clearly showing the individual cells. The intensity scale bar in $\mathbf{d}$ also applies to this image. 
dataset for iterative phase retrieval. The algorithm converges rapidly, typically requiring only $10-50$ iterations to reach its final solution. While the algorithm already works with two images, the use of more wavelengths usually provides superior results. When more wavelengths are used, the algorithm averages over more independent datasets, causing any noisy pixels in a single image to be less influential.

The multiwavelength phase retrieval algorithm results in a highquality image reconstruction, which is displayed in Figure $2 \mathrm{~d}$. Because the sample fills most of the field of view (FOV) and consists of dark structures on a bright illuminated background, the oversampling condition is not satisfied and support-based phase retrieval algorithms would not provide a solution in this situation. In contrast, our multiwavelength algorithm enables image reconstruction at instrument-limited resolution, clearly showing the individual cells as well as some subcellular features (Figure 2e). After the phase has been retrieved, all spectrally resolved images can be propagated to the object plane and averaged to improve the signal-to-noise ratio, resulting in an efficient use of the full-source spectrum. The resolution of this reconstruction is then determined by the imaging geometry and the weighted mean of the spectral bandwidth.

The main prerequisite of our multiwavelength phase retrieval approach is that the phase profile of the object has the same spatial structure for the spectral components used in the reconstruction. However, it should be stressed that the phase does not need to be constant as a function of wavelength: a known wavelength-dependent refractive index can be readily incorporated into the algorithm, as its incorporation requires only an additional refractive index-dependent multiplication factor upon propagation between different wavelength images. Therefore, our multiwavelength phase retrieval approach extends to dispersive samples and can be used for lensless imaging with short wavelengths. Wavelength-dependent differences in absorption result in different signal intensities for the respective images, while still providing a good-quality reconstruction. Only in the case where the phase images are qualitatively different at the different wavelengths, e.g., due to an elemental absorption edge between the wavelengths used, would the algorithm not converge. In this case, support-based iterative phase retrieval schemes can still provide image reconstruction, provided that the object meets the oversampling requirements for such algorithms to converge. ${ }^{7}$

\section{Lensless imaging in reflection}

In addition to transmission imaging, lensless imaging in a reflection geometry is important for many applications in nanoscience and technology. ${ }^{2,35}$ The need for a finite support is particularly challenging to satisfy for reflection geometries, ${ }^{36}$ which have therefore relied mostly on finite-beam-size methods such as ptychography. In contrast, our multiwavelength approach enables the imaging of large areas of extended samples in a reflection geometry (Figure $3 \mathrm{a}$ ). Figure $3 \mathrm{~b}$ shows a spectrally resolved Fresnel diffraction pattern, retrieved from a two-pulse scan in the reflection of a USAF 1951 test target. The reconstructed image obtained through multiwavelength phase retrieval is shown in Figure $3 \mathrm{c}$, demonstrating the ability to image large reflective samples without support requirements.

In a coherent diffractive imaging experiment, the achievable diffraction-limited resolution is determined by the wavelength of the light and the numerical aperture of the detection geometry (i.e., the size of the camera and the sample-to-camera distance). In the Fresnel geometry, the CCD pixel size also influences the final resolution. This resolution defines the distance between adjacent sampling points and the FOV is then determined by this distance multiplied by the number of pixels in each dimension. Therefore, the FOV is also wavelengthdependent. Consequently, when three diffraction patterns of an object are recorded at different wavelengths, the shorter wavelength pattern will have a slightly smaller FOV than the longer wavelength patterns. In the reconstruction, the FOV will therefore be limited by the shortest wavelength used. This limitation is illustrated in Figure $3 \mathrm{c}$, where the red dashed line indicates the FOV for the shortest wavelength. Outside this line, not all wavelengths contribute to the reconstruction, which results in aberrations.

In general, the iterative phase retrieval algorithm provides accurate phase information. If the diffraction comes from a pure surface reflection, this phase information can be used to reconstruct a height map of the sample. Figure $3 \mathrm{~d}$ displays a height map of the test target that has been retrieved in this way, clearly showing the $100 \mathrm{~nm}$ thick chromium lines deposited on a glass substrate. The phase retrieval algorithm already provides a height resolution of $\sim 20 \mathrm{~nm}$, which can be increased further by improving the signal-to-noise ratio in the measurement. In this sample, a small reflection from the sample's back surface was also present, resulting in an additional image that appears negative and shifted. Interference between these two surface images has some influence on the reconstructed phase, which would not be the case for a pure single surface reflection. Note that this measurement is of the optical path length, so that the refractive index of an immersion liquid or phase shifts due to partial reflection should be taken into account when calculating the geometrical height from the retrieved phase.

\section{Ultra-broadband XUV imaging}

To demonstrate that our two-pulse imaging approach extends to shorter wavelengths, we perform an imaging experiment with a HHG source. A schematic of the set-up is shown in Figure 4a: the pulse pair is produced in the near infrared with a split-wavefront interferometer in front of the HHG set-up, using quartz wedges to scan the time delay. This particular interferometer implementation is highly stable: it is nearly a common path and there are no mirrors in the set-up that reflect only one of the pulses. These properties eliminate most of the noise caused by vibrations, air flow and acoustics present in conventional Michelson interferometers. We use a $1^{\circ}$ wedge to scan the delay, mounted on a closed-loop piezo-stage with $5 \mathrm{~nm}$ resolution and a $500 \mu \mathrm{m}$ scan range. A $100 \mathrm{~nm}$ movement of the stage results in a $1.32 \mathrm{~nm}$ optical path length difference between the pulses, enabling scans with subnanometer step size.

A single wedge in the other interferometer arm introduces an angle between the beams. The lens (focal length $F$ ) that focuses the pulses into the HHG gas cell is placed at a distance $F$ behind the point where the beams cross. This geometry ensures that the two beams run parallel to each other behind the lens. Both beams focus near the end of the gas cell with a small transverse separation between the focal spots. Thus, ionization due to the first pulse does not influence the HHG process for the second (time-delayed) pulse, allowing linear Fourier transform scans at HHG frequencies. ${ }^{37}$ The HHG pulses overlap spatially at the imaging target due to their beam divergence. The focal length $F$ is $350 \mathrm{~mm}$ and the focal spot diameter is $40 \mu \mathrm{m}$ full width at half maximum. Harmonics are produced in xenon gas at $\sim 50$ mbar pressure in the gas cell, with an energy of $0.2 \mathrm{~mJ}$ in each $40 \mathrm{fs}$ duration pulse. Behind the cell, a differential pumping stage is used to prevent the re-absorption of the produced HHG. Scanning the time delay was performed by moving a wedge into one half of the beam, enabling scans with subnanometer position accuracy. 

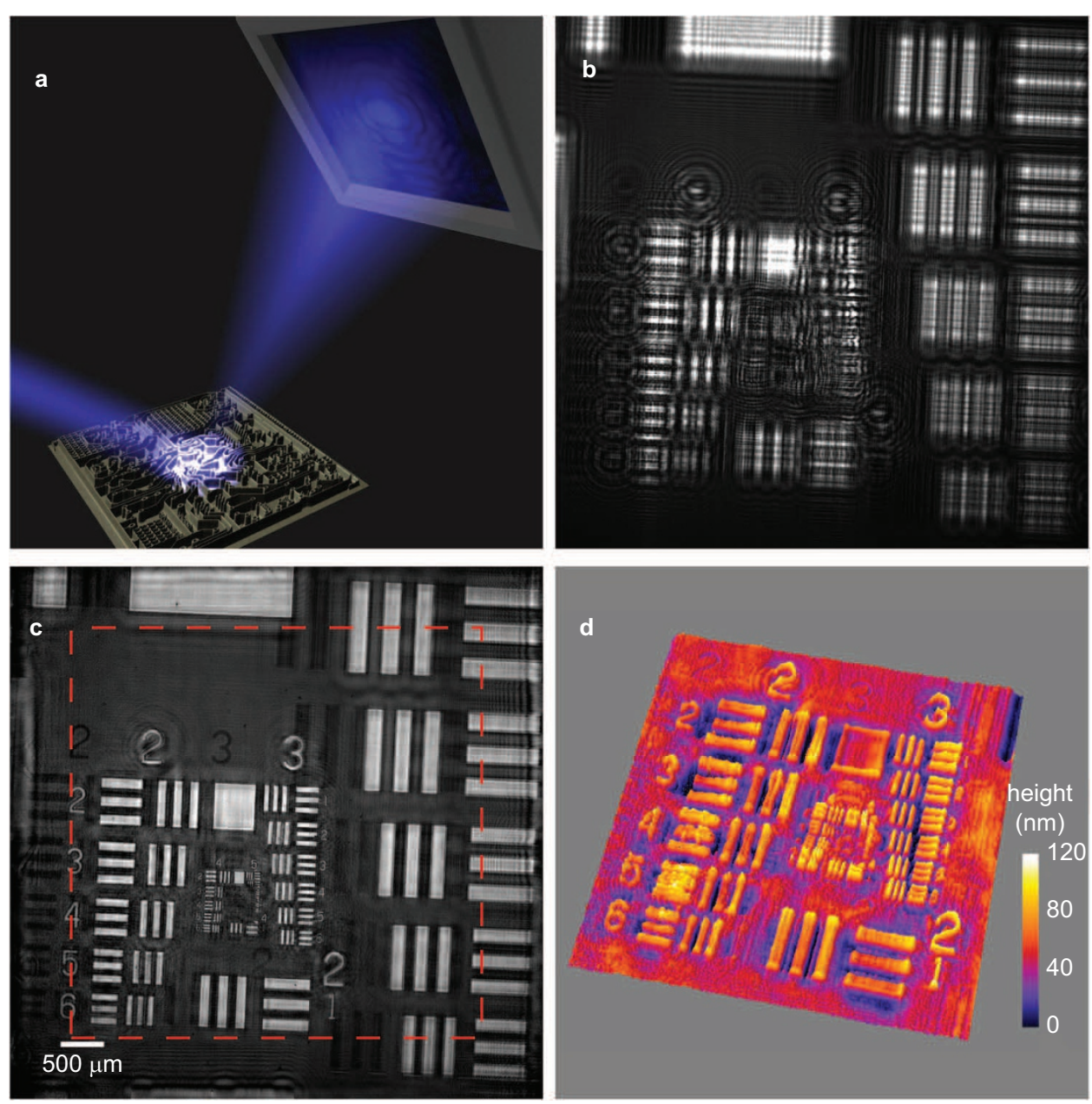

Figure 3 (a) Lensless imaging in a reflection geometry. (b) Quasimonochromatic Fresnel diffraction pattern of a 1951 USAF test target, recorded in a reflection geometry and obtained through a two-pulse Fourier-transform scan. (c) Resulting image after 25 iterations of the multiwavelength iterative phase retrieval, using diffraction patterns at five different wavelengths. The dark (negative) features are due to reflections from the sample's back surface. The dashed red line marks the area in which all wavelengths contribute to the reconstruction. (d) Reconstructed height map from part of the USAF target surface from b showing the chrome structures on the glass substrate. USAF, United States Air Force.

We use the full HHG flux directly to image a nickel grid, with $20 \mu \mathrm{m}$ wide bars, attached to a $300 \mathrm{~nm}$ thick aluminum foil. The $\mathrm{Al}$ foil reflects the fundamental beam while transmitting radiation at a wavelength below $80 \mathrm{~nm}$. In this experiment, the nickel grid is placed $40 \mathrm{~cm}$ behind the focus. The diffracted light is detected in a transmission geometry using an XUV-sensitive CCD camera (Andor Technology, Belfast, Northern Ireland) with $1024 \times 1024$ pixels, a pixel size of $13 \mu \mathrm{m}$ and a bit depth of 12 bits. This camera is placed $0.6 \mathrm{~m}$ behind the sample. In a typical Fourier transform scan, 512 diffraction images are recorded as a function of the time delay. Between consecutive images, the time interval is increased in steps of 44 attoseconds (corresponding to a physical step size of $13.2 \mathrm{~nm}$ ). At each time step, five frames are recorded with $0.3 \mathrm{~s}$ exposure times and averaged to improve the signal-to-noise ratio.

Figure $4 \mathrm{~b}$ displays a broadband diffraction pattern of the nickel grid recorded with the transmitted HHG spectrum. Figure 4c shows the HHG spectrum used in these imaging experiments, which is obtained from the two-pulse scan itself. Three harmonics are present, spanning a wavelength range between 47 and $63 \mathrm{~nm}$. Lower harmonics are not transmitted by the $\mathrm{Al}$ foil and the xenon gas, while the HHG phasematching cutoff is near harmonic 17. For the HHG phase retrieval, we use images at harmonics 13, 15 and 17 (center wavelengths $62 \mathrm{~nm}$,
$53 \mathrm{~nm}$ and $47 \mathrm{~nm}$, respectively) as input for the multiwavelength phase retrieval algorithm. At each harmonic, we filter out a spectral bandwidth of $1.5 \mathrm{~nm}$, which suffices to achieve diffraction-limited resolution in this experiment. Typically, only 10 iterations of the algorithm are required for stable convergence.

Due to the transverse displacement between the two HHG pulses, the recorded data contain two displaced copies of the object's diffraction pattern. This factor can be accounted for by incorporating an additional deconvolution step in the image reconstruction procedure, as the image at each wavelength can be interpreted as the convolution between a single diffraction pattern of the object and a pair of Dirac delta functions. The distance between these Dirac delta functions is calculated from the imaging geometry and the initial separation between the focal spots in the gas cell. The single diffraction patterns can then be retrieved from the spectrally resolved images by deconvolution. The resulting diffraction patterns can then be used in the multiwavelength phase retrieval algorithm. Small angular deviations between the two beams can also be handled, enabling two-pulse imaging even with non-collinear beams from a split-wavefront interferometer. ${ }^{38,39}$

The resulting reconstructed image of the $\mathrm{Ni}$ grid is shown in Figure $4 \mathrm{~d}$ and $4 \mathrm{e}$. To quantify the image resolution, the image intensity 

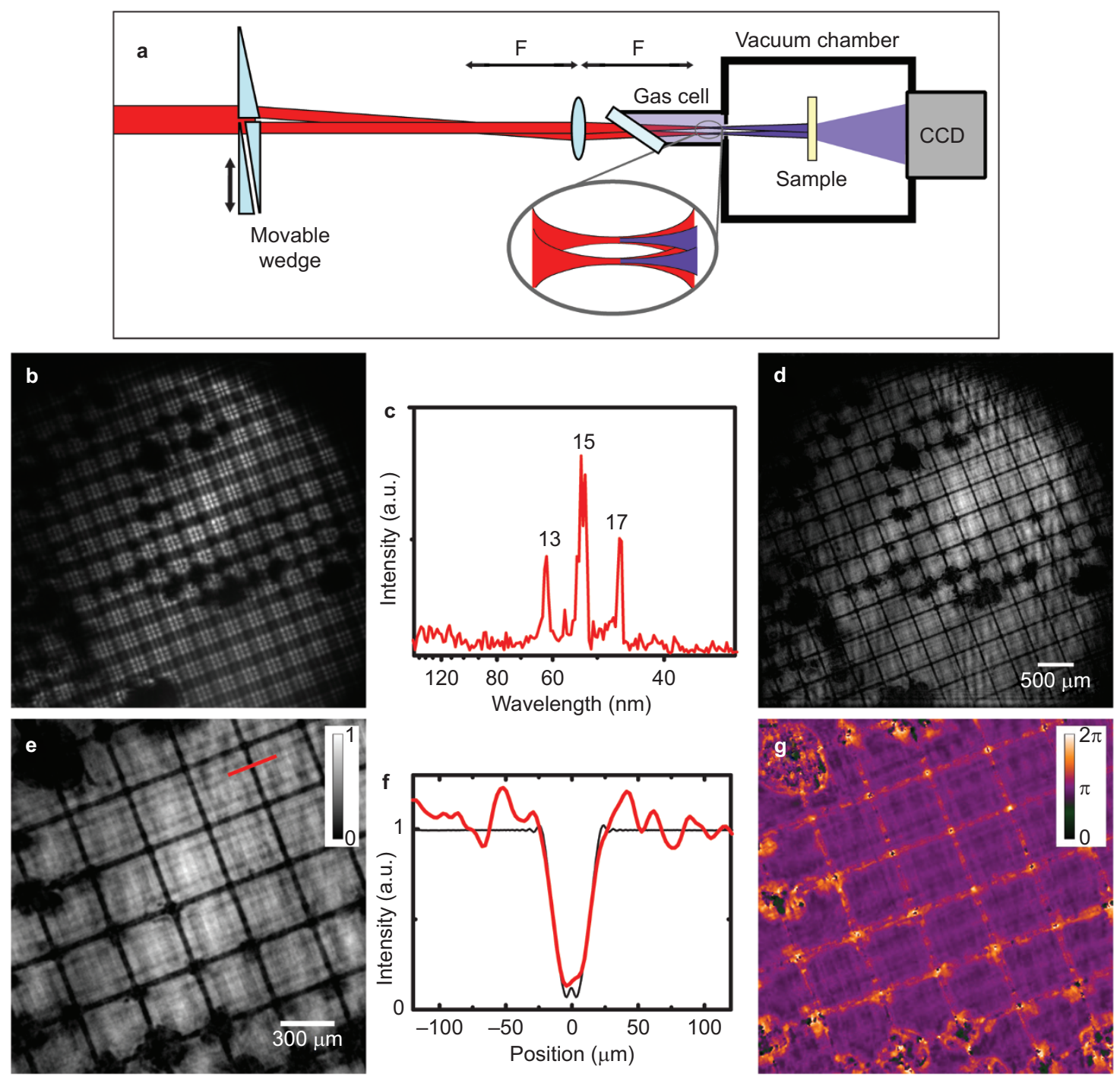

Figure 4 Lensless imaging with ultra-broadband extreme-ultraviolet radiation. (a) Schematic of the set-up for two-pulse imaging with high-harmonic radiation. A variable time delay is introduced by inserting a wedge into the beam (note that this drawing is only for schematic purposes: the actual orientation of the wedges is rotated by $90^{\circ}$ with respect to this drawing). (b) Broadband diffraction pattern of a nickel grid on a $300 \mathrm{~nm}$ thick Al foil, recorded using harmonics 13, 15 and 17. (c) Spectrum extracted from the two-pulse Fourier transform scan. (d) Retrieved image of the Ni grid at $47 \mathrm{~nm}$ wavelength, using the multiwavelength phase retrieval algorithm. The regular features of the grid are clearly visible, as are several damage spots induced by intense laser irradiation in earlier experiments. (e) Enlarged view of part of the image in d, showing the $20 \mu \mathrm{m}$ wide grid lines. (f) Cross-section of the intensity along the red bar in e (red trace), together with a simulated curve of a square bar convolved with the expected diffraction limited point-spread function (black trace). (g) Phase image of the grid retrieved by the multiwavelength phase retrieval algorithm.

across one of the nickel bars is plotted in Figure $4 \mathrm{f}$. This cross-section is compared to a simulation of the known shape of the bar convolved with a sinc-shaped point-spread function corresponding to the diffraction-limited half-pitch resolution of $6.7 \mu \mathrm{m}$. Good agreement is obtained, from which we deduce that the retrieved image resolution is near the diffraction limit for the sample-camera distance used. A small artifact from imperfect deconvolution can be observed in the form of a shadow image (with an intensity of a few percent) on both sides of the main image. Furthermore, some subtle vertical interference fringes are observed in the reconstructed image in Figure 4d, mainly at the right edge of the beam. These fringes are likely caused by imperfections in the incident XUV wavefront. In addition to the regular Ni grid, several dark damage spots are observed, which were caused in earlier experiments with intense laser pulses that partially melted the nickel structures. In addition to the reconstructed intensity image, the retrieved phase profile at $47 \mathrm{~nm}$ wavelength is shown in Figure $4 \mathrm{~g}$. A relatively constant phase is obtained for the gaps between the nickel bars, while the phase at the position of the bars shows large variations. This observation is explained by the negligible light intensities at the locations of the bars, which are $5 \mu \mathrm{m}$ thick and do not transmit XUV radiation. Therefore, the phase at these positions is undefined. While the diffraction limit in the current proof-of-concept experiment was relatively low due to geometrical constraints, a much higher resolution can readily be achieved in Fresnel diffractive imaging by illuminating the sample with a curved wavefront, ${ }^{11,29}$ which is fully compatible with our two-pulse imaging approach.

\section{CONCLUSIONS}

In summary, we have presented two-pulse imaging as a method that enables lensless microscopy with ultra-broadband light sources. The 
full spectrum is efficiently used for imaging, making this approach well suited for XUV imaging with compact, low-flux, broadband sources such as table-top HHG systems.

In addition, we have introduced a new approach to robust phase retrieval for lensless imaging in the Fresnel regime, based on the use of diffraction patterns at multiple wavelengths in an iterative phase retrieval scheme. This method provides stable and rapid convergence even for complex samples, without requiring any support constraints or localized illumination. This multiwavelength phase retrieval approach can readily be used with the spectrally resolved data obtained through two-pulse imaging and we have demonstrated its use in both transmission and reflection.

We find that two-pulse imaging enables robust and accurate lensless imaging with broadband and unstable spectra, without a priori knowledge of the spectrum for image reconstruction. This situation is often encountered with HHG sources, where the efficient use of the available photon flux is essential for practical imaging applications. We have performed numerical simulations to study the influence of intensity and timing variations in greater detail (Supplementary Fig. S4 and Supplementary Information) and the simulations indicate that goodquality images can be obtained even in the presence of significant noise. From our measurements, we find a pulse delay stability of $8 \mathrm{~nm}$ without active stabilization (Supplementary Fig. S3 and Supplementary Information), which is sufficient for imaging at wavelengths down to $16 \mathrm{~nm}$. With additional stabilization measures, ${ }^{40}$ an extension to the soft$\mathrm{X}$-ray domain should be feasible. We therefore foresee that two-pulse imaging will find widespread application in the development of compact table-top HHG-based microscopes, thus providing new possibilities for, e.g., structural biology and nanotechnology by enabling label-free ultrahigh-resolution microscopy in a laboratory-scale environment.

\section{ACKNOWLEDGMENTS}

The authors thank PS Carney for insightful discussions and ML Groot for providing access to her Ti:Sapphire amplifier system. This work is financed in part by an NWO-groot investment grant of the Netherlands Organisation for Scientific Research (NWO) and Laserlab Europe (JRA Bioptichal). SW acknowledges support from NWO Veni grant 680-47-402.

1 Chapman HN, Nugent KA. Coherent lensless X-ray imaging. Nat Photonics 2010; 4: 833-839.

2 Sakdinawat A, Attwood D. Nanoscale X-ray imaging. Nat Photonics 2010; 4: 840-848.

3 Zheng G, Lee SA, Antebi Y, Elowitz MB, Yang C. The ePetri dish, an on-chip cell imaging platform based on subpixel perspective sweeping microscopy (SPSM). Proc Natl Acad Sci USA 2011; 108: 16889-16894.

4 Isikman SO, Bishara W, Mavandadi S, Yu FW, Feng S et al. Lens-free optical tomographic microscope with a large imaging volume on a chip. Proc Natl Acad Sci USA 2011; 108: 7296-7301.

5 Miao J, Charalambous P, Kirz J, Sayre D. Extending the methodology of X-ray crystallography to allow imaging of micrometre-sized non-crystalline specimens. Nature 1999; 400: 342-344.

6 McNulty I, Kirz J, Jacobsen C, Anderson EH, Howells MR et al. High-resolution imaging by Fourier transform X-ray holography. Science 1992; 256: 1009-1012.

7 Marchesini S. A unified evaluation of iterative projection algorithms for phase retrieval. Rev Sci Instrum 2007; 78: 011301.

8 Bruck YM, Sodin LG. On the ambiguity of the image reconstruction problem. Opt Commun 1979; 30: 304-308.

9 Marchesini S, He H, Chapman HN, Hau-Riege SP, Noy A et al. X-ray image reconstruction from a diffraction pattern alone. Phys Rev B 2003; 68: 140101(R).

10 FienupJR. Reconstruction of a complex-valued object from the modulus of its Fourier transform using a support constraint. J Opt Soc Am A 1987; 4: 118-123.

11 Abbey B, Nugent KA, Williams GJ, Clark JN, Peele AG et al. Keyhole coherent diffractive imaging. Nat Phys 2008; 4: 394-398.

12 Thibault P, Dierolf M, Menzel A, Bunk O, David C et al. High-resolution scanning x-ray diffraction microscopy. Science 2008; 321: 379-382.
13 Faulkner H, Rodenburg J. Movable aperture lensless transmission microscopy: a novel phase retrieval algorithm. Phys Rev Lett 2004; 93: 023903.

14 Marchesini S, Boutet S, Sakdinawat AE, Bogan MJ, Bajt S et al. Massively parallel Xray holography. Nat Photonics 2008; 2: 560-563.

15 Chen MC, Arpin P, Popmintchev T, Gerrity M, Zhang B et al. Bright, coherent, ultrafast soft X-ray harmonics spanning the water window from a tabletop light source. Phys Rev Lett 2010; 105: 173901

16 Popmintchev T, Chen MC, Popmintchev D, Arpin P, Brown S et al. Bright coherent ultrahigh harmonics in the keV X-ray regime from mid-infrared femtosecond lasers. Science 2012; 336: 1287-1291.

17 Sandberg RL, Song C, Wachulak PW, Raymondson DA, Paul A et al. High numerical aperture tabletop soft X-ray diffraction microscopy with 70-nm resolution. Proc Nat/ Acad Sci USA 2008; 105: 24-27.

18 Sandberg RL, Raymondson DA, La-O-Vorakiat C, Paul A, Raines KS et al. Tabletop soft-X-ray Fourier transform holography with $50 \mathrm{~nm}$ resolution. Opt Lett 2009; 34 $1618-1620$.

19 Morlens AS, Gautier J, Rey G, Zeitoun P, Caumes JP et al. Submicrometer digital inline holographic microscopy at $32 \mathrm{~nm}$ with high-order harmonics. Opt Lett 2006; 31: 3095-3097.

20 Ravasio A, Gauthier D, Maia FR, Billon M, Caumes JP et al. Single-shot diffractive imaging with a table-top femtosecond soft X-ray laser-harmonics source. Phys Rev Lett 2009; 103: 028104.

21 Wieland M, Frueke R, Wilhein T, Spielmann C, Pohl M et al. Submicron extreme ultraviolet imaging using high-harmonic radiation. Appl Phys Lett 2002; 81: 2520-2522.

22 Seaberg MD, Adams DE, Townsend EL, Raymondson DA, Schlotter WF et al. Ultrahigh $22 \mathrm{~nm}$ resolution coherent diffractive imaging using a desktop $13 \mathrm{~nm}$ high harmonic source. Opt Express 2011; 19: 22470-22479.

23 Chen B, Dilanian RA, Teichmann S, Abbey B, Peele AG et al. Multiple wavelength diffractive imaging. Phys Rev A 2009; 79: 023809.

24 Parsons AD, Chapman RT, Mills B, Butcher TJ, Frey JG et al. $90 \mathrm{~nm}$ resolution reconstruction from a polychromatic signal using monochromatic phase retrieval techniques. In: Proceedings of the European Conference on Lasers and ElectroOptics (CLEO/Europe-EQEC 2011); 22-26 May 2011; Munich, Germany. Optical Society of America: Washington, DC, USA, 2011, CD_P35.

25 Abbey B, Whitehead LW, Quiney HM, Vine DJ, Cadenazzi GA et al. Lensless imaging using broadband X-ray sources. Nat Photonics 2011; 5: 420-424.

26 Becker ED, Farrar TC. Fourier transform spectroscopy. Science 1972; 178: 361-368.

27 Yablon AD. Multi-wavelength optical fiber refractive index profiling by spatially resolved Fourier transform spectroscopy. J Light Technol 2010; 28: 360-364.

28 Ferreras Paz V, Peterhänsel S, Frenner K, Osten W. Solving the inverse grating problem by white light interference Fourier scatterometry. Light Sci App/ 2012; 1: e36; doi:10.1038/lsa.2012.36

29 Williams GJ, Quiney HM, Dhal BB, Tran CQ, Nugent KA et al. Fresnel coherent diffractive imaging. Phys Rev Lett 2006; 97: 025506.

30 Quiney HM, Nugent KA, Peele AG. Iterative image reconstruction algorithms using wave-front intensity and phase variation. Opt Lett 2005; 30: 1638-1640.

31 Latychevskaia T, Fink HW. Solution to the twin image problem in holography. Phys Rev Lett 2007; 98: 233901.

32 Allen LJ, Oxley MP. Phase retrieval from series of images obtained by defocus variation. Opt Commun 2001; 199: 65-75.

33 Greenbaum A, Ozcan A. Maskless imaging of dense samples using pixel superresolution based multi-height lensfree on-chip microscopy. Opt Express 2012; 20: 3129-3143.

34 Zhang B, Seaberg MD, Adams DE, Gardner DF, Shanblatt ER et al. Full field tabletop EUV coherent diffractive imaging in a transmission geometry. Opt Express 2013; 21: 21970-21980

35 La-O-Vorakiat C, Siemens M, Murnane MM, Kapteyn HC, Mathias S et al. Ultrafast demagnetization dynamics at the $M$ edges of magnetic elements observed using a tabletop high-harmonic soft X-ray source. Phys Rev Lett 2009; 103: 257402.

36 Roy S, Parks D, Seu KA, Su R, Turner JJ et al. Lensless X-ray imaging in reflection geometry. Nat Photonics 2011; 5: 243-245.

37 Kovačev M, Fomichev SV, Priori E, Mairesse Y, Merdji H et al. Extreme ultraviolet Fouriertransform spectroscopy with high order harmonics. Phys Rev Lett 2005; 95: 223903.

38 de Oliveira N, Roudjane M, Joyeux D, Phalippou D, Rodier JC et al. High-resolution broad-bandwidth Fourier-transform absorption spectroscopy in the VUV range down to 40 nm. Nat Photonics 2011; 5: 149-153.

39 Günther CM, Pfau B, Mitzner R, Siemer B, Roling S et al. Sequential femtosecond Xray imaging. Nat Photonics 2011; 5: 99-102.

40 Brida D, Manzoni C, Cerullo G. Phase-locked pulses for two-dimensional spectroscopy by a birefringent delay line. Opt Lett 2012; 37: 3027-3029.

This work is licensed under a Creative Commons AttributionNonCommercial-NoDerivs Works 3.0 Unported license. To view a copy of isit http://creativecommons.org/licenses/by-nc-nd/3.0 